\title{
Pseudohypoparathyroidism and Cardiomyopathy: A Case Report with a New Perspective on the Cardiovascular-Endocrine Axis with Respect to Calcium Homeostasis
}

\author{
Anum Fasih \\ Department of Family Medicine, University of Illinois, College of Medicine, Peoria, IL, USA
}

Received: $11 / 11 / 2018$

Accepted: $14 / 01 / 2019$

Published: 15/02/2019

\begin{abstract}
How to cite this article: Fasih A. Pseudohypoparathyroidism and cardiomyopathy: a case report with a new perspective on the cardiovascular-endocrine
\end{abstract} axis with respect to calcium homeostasis. EJCRIM 2019;6: doi:10.12890/2019_000993.

Conflicts of Interests: The Authors declare that there are no competing interests.

This article is licensed under a Commons Attribution Non-Commercial 4.0 License

\section{ABSTRACT}

Pseudohypoparathyroidism encompasses a group of heterogeneous disorders defined by targeted organ unresponsiveness to parathyroid hormone (PTH). With an estimated prevalence of approximately 0.79 per 100,000 , this is an extremely rare group of genetic disorders. The documented phenotypic manifestations and complications of this condition include skeletal abnormalities, thyroid-stimulating hormone (TSH) resistance, growth hormone deficiencies and abnormalities in gonadotropic hormones. However, the cardiovascular effects of this condition have never been studied. To the knowledge of the writer, this is the first report of pseudohypoparathyroidism resulting in profound hypocalcaemia leading to heart failure in a previously healthy 22-year-old man with no known cardiac history. Following treatment with calcium replacement therapy, the patient's cardiac function improved dramatically, demonstrating the seminal role played by serum calcium in myocardial contractility and its influence on cardiac function.

\section{LEARNING POINTS}

- In patients with heart failure, serum calcium must be measured, especially if the underlying aetiology of heart failure is unclear.

- In patients with heart failure, correction of calcium levels is an important aspect of management.

- Patients with pseudohypoparathyroidism should undergo cardiac evaluation, especially in the presence of symptoms such as peripheral oedema and dyspnoea on exertion.

\section{KEYWORDS}

Pseudohypoparathyroidism, hypocalcemia, heart failure, case report

\section{INTRODUCTION}

Pseudohypoparathyroidism (PHP) refers to a group of extremely rare heterogeneous disorders defined by targeted organ (kidney and bone) unresponsiveness to parathyroid hormone $(\mathrm{PTH})^{[1]}$. The prevalence of PHP is estimated to be approximately 0.79 per $100,000^{[2]}$. The phenotypic manifestations and complications of this condition include skeletal abnormalities, thyroid-stimulating hormone (TSH) resistance, growth hormone deficiencies and abnormalities in gonadotrophic hormone ${ }^{[2]}$. However, the cardiovascular effects of this condition have not been described. The first reported case of PHP resulting in profound hypocalcaemia leading to heart failure is described below. It is widely accepted that the generation, modulation and termination of intracellular calcium transients underlies myocardial excitation- 
contraction coupling ${ }^{[3]}$, making calcium an electrolyte critical to the functional integrity of the heart. However, monitoring and regulation of serum calcium is not an important part of the management of heart failure.

\section{CASE PRESENTATION}

A 22-year-old man presented to the Emergency Department (ED) with complaints of dyspnoea and severe bilateral lower extremity pain following intense physical exertion while competing in a body-building competition earlier in the day. Aside from a diagnosis of PHP, the patient had been fairly healthy; he did not have a history of diabetes, hypertension, hyperlipidaemia or obesity, did not smoke or use illicit drugs, and exercised regularly. The patient did not have any phenotypic manifestations of PHP and was of average height. On arrival at the ED, the patient's respiratory rate was in the high 20 's, and his oxygen saturation was $88-89 \%$ on room air. A CT angiogram was negative for a pulmonary embolism. An x-ray showed an enlarged cardiac silhouette and signs of pulmonary oedema. Interestingly, an echocardiogram showed severe global hypokinesis and severely decreased left ventricular systolic function with an ejection fraction of $25 \%$. The patient was placed on bilevel positive airway pressure (BiPAP) and admitted to an intensive care unit, following which he was transferred to the ICU for worsening respiratory status.

Initial investigations revealed an elevated CK, indicative of severe rhabdomyolysis that was likely a consequence of intense physical activity during the body-building competition. The rhabdomyolysis resulted in compartment syndrome, which had caused the patient's excruciating lower extremity pain. The patient's renal function was monitored closely and remained normal at all times.

As is known to happen ${ }^{[4]}$, the rhabdomyolysis also resulted in a severe drop in a pre-existing low serum calcium level. The patient had chronic baseline hypocalcaemia due to PHP, a condition with which he was diagnosed at age 17. Review of previous records revealed a serum calcium concentration of 6.5-7.5 mg/dl (normal range 8.5-10.2) over the years. This, taken together with an elevated phosphate and high PTH ranging between 425 and 550 pg/ml (normal range 15-65) supported the diagnosis of PHP, most likely type 1 b given the absence of the phenotypic abnormalities of Albright hereditary osteodystrophy. Prior to admission, the patient was not on any medications, and did not admit to use of nutritional supplements.

During this admission, the patient was found to have a presenting ionized serum calcium of $3.3 \mathrm{mg} / \mathrm{dl}$ (normal range 4.8-5.2). Laboratory investigation also showed high serum phosphate $(5.8 \mathrm{mg} / \mathrm{dl})$ and PTH $(325 \mathrm{pg} / \mathrm{ml})$ levels. A 24-hour urine calcium was also found to be elevated at $728 \mathrm{mg}$ (range 50-350). In addition to diuresis for fluid overload, the patient was treated with intravenous and oral calcium replacement therapy to target hypocalcaemia. By the time of discharge, ionized serum calcium levels had reached near normal concentrations (4.7 mg/dl), and cardiac function had improved, with an increase in the ejection fraction to $45 \%$ as demonstrated by a repeat echocardiogram. A cardiac MRI was also performed in order to exclude other aetiologies of heart failure, and showed no evidence of an infiltrative process. Other negative investigations included a full autoimmune, infectious and toxicological work-up.

After a prolonged stay in hospital during which the patient experienced complications of compartment syndrome which required numerous surgical interventions, he was discharged, with his cardiac function near normal and his respiratory measurements stable on room air.

Interestingly, a review of the patient's medical records revealed several previous admissions, once at age 10 and twice at age 17, with a chief complaint of perioral numbness and cramping in his jaw. During these admissions, the patient was found to have low serum calcium and was treated with calcium replacement, leading to resolution of his symptoms. Furthermore, the records also documented several outpatient visits with complaints of 'heart failure-like' symptoms, that is, dyspnoea and lower extremity swelling which almost always followed periods of intense physical activity. The patient was treated with diuretics, which helped alleviate his symptoms.

\section{DISCUSSION}

The sequence of events in this case follows a logical pattern of pathology; in a patient with known PHP resulting in low baseline serum calcium levels, intensive physical activity led to a further reduction in serum calcium levels which caused a decline in cardiac contractility and function, which manifested as dyspnoea and lower extremity oedema, cardinal signs and symptoms of heart failure.

The generation, modulation and termination of the intracellular calcium transients underlies myocardial excitation-contraction coupling ${ }^{[3]}$, making calcium an electrolyte critical to the functional integrity of the heart.

One study observed the effects of calcium replacement on myocardial function in six asymptomatic patients with hypocalcaemia complicating surgical hypoparathyroidism. This study found that 3 months after normalization of calcium by oral therapy, both maximum cardiac output and maximum heart rate demonstrated a statistically significant increase during peak exercise, thus concluding that hypocalcaemia impairs cardiac performance, but that this impairment is reversible with calcium replacement ${ }^{[5]}$. Several case reports have demonstrated similar findings; that is, hypocalcaemia is a reversible and, more importantly, easily treatable cause of heart failure ${ }^{[6-10]}$.

Nonetheless, literature on the integral role that calcium plays in ensuring optimum myocardial function is severely lacking. The Heart Failure Society of America outlines the management of serum potassium and magnesium levels for the management of acute exacerbation of heart 
failure ${ }^{[11]}$. However, very little is said about the role of serum calcium in the pathophysiology of impaired myocardial contractility and how to address this clinically. For the long-term management of heart failure, the American College of Cardiology/American Heart Association Taskforce issued in its Practice Guidelines Report its recommendations regarding the use of cardiac medications ${ }^{[12]}$. Although the use of calcium channel blockers was generally advised against, the normalization of calcium levels for those with hypocalcaemia was not addressed.

\section{REFERENCES}

1. Chase LR, Melson GL, Aurbach GD. Pseudohypoparathyroidism: defective excretion of 3',5'-AMP in response to parathyroid hormone. J Clin Invest 1969;48:1832.

2. Mantovani G. Pseudohypoparathyroidism: diagnosis and treatment. J Clin Endocrinol Metab 2011;96:3020-3030.

3. Smith TW, Morgan JP. Excitation-contraction coupling in myocardium. Uptodate.com. Available from https://www.uptodate.com/contents/excitation-contraction-coupling-inmyocardium?topicRef=833\&source=see_link\#H7 (accessed 15 August 2018).

4. Akmal M, Bishop JE, Telfer N, Norman AW, Massry SG. Hypocalcemia and hypercalcemia in patients with rhabdomyolysis with and without acute renal failure. J Clin Endocrinol Metab 1986;63:137.

5. Wong CK, Lau CP, Cheng CH, Leung WH, Freedman B. Hypocalcemic myocardial dysfunction: short- and long-term improvement with calcium replacement. Am Heart J 1990;120:381.

6. Shinoda T, Aizawa T, Shirota T. Exacerbation of latent heart failure by mild hypocalcemia after parathyroidectomy in a long-term hemodialysis patient. Nephron $1992 ; 60: 482$.

7. Kazmi AS, Wall BM. Reversible congestive heart failure related to profound hypocalcemia secondary to hypoparathyroidism. Am J Med Sci 2007;333:226.

8. Levine SN, Rheams CN. Hypocalcemic heart failure. Am J Med 1985;78(6 Pt 1):1033.

9. Kudoh C, Tanaka S, Marusaki S. Hypocalcemic cardiomyopathy in a patient with idiopathic hypoparathyroidism. Intern Med 1992;31:561.

10. Brunvand L, Hågå P, Tangsrud SE, Haug E. Congestive heart failure caused by vitamin D deficiency? Acta Paediatr 1995;84:106.

11. Lindenfeld J, Albert NM, Boehmer JP, et al.; Heart Failure Society of America. HFSA 2010 Comprehensive Heart Failure Practice Guideline. J Card Fail 2010;16(6):e1-194.

12. Yancy CW, Jessup M, Bozkurt B, et al. 2013 ACCF/AHA guideline for the management of heart failure: a report of the American College of Cardiology Foundation/American Heart Association Task Force on Practice Guidelines. J Am Coll Cardiol 2013;62:e147. 